Full length article

\title{
A full scope web accessibility evaluation procedure proposal based on Iberian eHealth accessibility compliance
}

\author{
José Martins*, Ramiro Gonçalves, Frederico Branco \\ University of Trás-os-Montes e Alto Douro, INESC TEC and UTAD, Vila Real, Portugal
}

\section{A R T I C L E I N F O}

\section{Article history:}

Received 30 June 2016

Received in revised form

29 November 2016

Accepted 5 December 2016

Available online 10 December 2016

\section{Keywords:}

Web accessibility

Web usability

Evaluation procedure

eHealth

Iberian Peninsula

Health care organisations

\begin{abstract}
A B S T R A C T
Internet is becoming one of the most adopted technologies of all times and one of its particular uses concerns the public health issues. The search for health related information and the exchange of experiences and opinions on symptoms and treatments is one of the main activities associated with eHealth websites, hence the need for these websites to be accessible to everybody, including those with some sort of disability. Nevertheless, when assessing the level of the WCAG 2.0 compliance of Iberian eHealth websites, the results achieved during a two stage, one year apart, evaluation indicated that these websites were definitely not accessible. By adding this finding to other similar results achieved by means of similar researches we believe that a new full scope Web accessibility and usability evaluation procedure was needed and is now presented. The referred proposal aims at creating a basis for both organisations and Web developers to understand how to perform an adequate assessment of their websites.
\end{abstract}

(๑) 2016 Elsevier Ltd. All rights reserved.

\section{Introduction}

As Internet becomes a common detail of citizens' everyday life, it also aggregates some of their necessities and wishes. One of the most relevant issues for the majority of the population is their health and the maintenance of a considerable degree of quality in their lives. The convergence of the two arguments has led to a shift in the search for health-related information that resulted on users focusing their attention on the Internet as one of the main repositories for that type of knowledge. The figures behind this evolution indicate that for more than $75 \%$ of the Europeans (EC., 2014) and over 65\% of all the United States adults (PRC, 2013) this fact is true. This can be extremely significant and act as an indicator that the existing online health information (eHealth) should constantly be of high quality, easy to access and simple to understand.

According to Kelly, Ziebland, and Jenkinson (2015) and Naoui and Zaiem (2015), the current websites and Web platforms that publish health related information are no longer just information repositories. These are used as places where users share

\footnotetext{
* Corresponding author.

E-mail addresses: jmartins@utad.pt (J. Martins), ramiro@utad.pt (R. Gonçalves), fbranco@utad.pt (F. Branco).
}

experiences, find support and advice for their issues. Hence the need for both Web developers and health professionals to not only understand the impact the health related content might have on the life of users, but also to acknowledge the need for the referred content to be usable and accessible to all the users, including those with some sort of disability (Klein, Bolfing, \& Riesch, 2014).

Despite both the relevance of "eHealth" and "Web accessibility \& usability" topics and associated scientific literature, as far as we know there isn't an in-depth analysis to the eHealth websites accessibility compliance levels, particularly one that allows to perceive its timeline evolution. The same fault exists when analysing the existing literature on methodologies or full scope procedures proposals that indicate how to perform an adequate Web accessibility and usability assessment (Martins et al., 2015; Martins et al., 2016).

The goal of the present research has been to analyse the Iberian eHealth websites in order to assess their levels of compliance with accessibility and usability guidelines and standards. This perspective has allowed a characterisation on how accessible is health information to citizens from both Portugal and Spain. Besides this goal, the research also has aimed on reaching a proposal for a full scope Web accessibility and usability evaluation procedure that includes both automatic and manual activities.

The manuscript is organised in five sections, starting from an over-the-top characterisation of the research project scope. The 
2nd section introduces a theoretical characterisation of all the concepts associated with the project. A characterisation of the Iberian eHealth websites timeline evaluation is presented in section 3 and followed by the proposal for a full scope Web accessibility and usability evaluation procedure. The manuscript is concluded with a Conclusions section.

\section{The conceptual framework}

\section{1. eHealth services}

As organisations immerse themselves in the existing Digital Society, the public availability of a corporate website tends to become a place where one cannot only perceive information directly related to the organisations' core, but also related to the organisations' field of activity (Á. Rocha, 2012). From a more commercial perspective, a corporate website can be considered to be the first interaction point between an organisation and its customers, thus the need to develop high quality websites and inherent content (Leite, Gonçalves, Teixeira, \& Rocha, 2014). According to Hwang, McMillan, and Lee (2003), when a corporation assumes a public online presence this should represent a strategic approach directed at communicating with their audiences, spreading their corporate image and, in parallel, displaying their services. This same argument is defended by Hakim and Deswindi (2015) when arguing that health care institutions websites are extremely important as a strategic tool for diminishing the distance that, so many times, exists between themselves and their customers/patients.

In a society eager for information, Internet users (patients) tend to create expectations regarding the access to eHealth (Eysenbach, 2001), that in this case can be translated in the desire to access high quality health information, better health expertise and medical services, cutting-edge treatments and health related support given in a more personalised manner (Domenichiello, 2015). From a business standpoint, the referred websites will increase the institutions' chances of being caught during their users raid of Internet search, hence extrapolating their chances on seizing new potential customers and triggering their existing customers' loyalty.

When analysing patients health care websites usage patterns, one can easily acknowledge that they are gaining abilities and needs towards understanding not only how to identify certain symptoms associated with some health issues, but also to understand what their chosen hospital can offer in terms of services and available experts (Huang, Liu, \& Wang, 2014). In the same line, and considering the research of de Haydu, Eleswarapu, Dabaja, and Duke (2015), eHealth platforms, particularly those directly supported by health care institutions, should serve as a repository for trustworthy health information that might be of some interest to patients. Nevertheless, there are still several institutions which continue to implement policies only directed at delivering a good medical service to the public, diminishing the attention given to create and provide useful and accurate health information, and allowing all citizens (including those with some sort of disability), to receive some initial diagnosis and have their doubts cleared (Raji, Mahmud, Tap, \& Abubakar, 2014). A straightforward engagement of both patients and their families to health related information and Web based systems will act as an element which will help at increasing the overall health care services performance, but also at establishing better and more solid relationships between medical experts and patients (Ralston et al., 2007).

Lin, Wittevrongel, Moore, Beaty, and Ross (2005) and Schenker and London (2015) argue that the availability of a website that provides insights on the quality ratings of a given hospital, its treatment policies, specialties and protocols, and its patients' decision aid mechanisms, will have a very significant impact on the patients' decisions towards seeking care or undergo a certain medical procedure. Thus, complying also with Raji et al. (2014), in order to be useful to their patients, hospitals' websites should adopt an easy to use, aesthetically attractive and, most of all, rich and accurate direct health care information.

With the above in mind, the requirement for a hospital website to be able to attract patients and allow them to search for health information, track their health record, make appointments and communicate with health services providers, it is clear that the referred websites must be easy to use and accessible to all (Robeznieks, 2011; Snyder, Ornes, \& Paulson, 2014).

The importance of high quality hospital websites, ease of use and accessibility has been in the agenda of several authors and organisations, who argue that, despite the notorious efforts in bringing the institutions to the Web, there is still a long way to go in order for the referred websites to be easily usable and accessed by everybody (Noh, Jeong, You, Moon, \& Kang, 2015; Silvestre, Tomlinson-Hansen, Fosnot, \& Taylor, 2014; Snyder et al., 2014; UN., 2014).

\subsection{Accessible websites}

As the current number of European citizens with some sort of disability reaches the 80 million mark, the European Union Agency for Fundamental Rights publicly highlights the need to be granted the same rights to both disabled and non-disabled citizens in what concerns integration within society, and the use of any product or service. This effort of equality should be even more considerable when perceiving all available ICT supported content, including the one that is only available online (FRA., 2015). Information and communication technologies are even more important when we analyse them as tools that give active support to users with functional limitations, hence allowing them a more complete society integration (Sánchez-Gordón \& Moreno, 2014). Even though the importance of having a universal ICT, and its inherent return in terms of life quality and integration is acknowledged, this will only be a reality when these technologies become accessible to all users, no matter of their disabilities or impairments (Gonçalves, Martins, Branco, \& Barroso, 2013; Wenner, 2008).

The Web, probably the most relevant ICT available to users, holds a role of extreme importance to both individuals and organisations, mainly because it allows the simple and linear establishment of virtual connections with positive return to both scopes (Sigala \& Chalkiti, 2014). Hence, the existence of criteria that support a universal access to the Web represents a clear necessity and the reasoning behind the Web accessibility concept.

As Fernandes, Costa, Duarte, and Carriço (2012) argue, a more accessible Web means that it can be used by all the users regardless of their limitations. This concept requests the absence of barriers that limit the users' interaction with the existing content, thus the mindset of a website which "excludes" users cannot be considered accessible. The ability of a given Web content to be accessible to all the users, including those with some sort of disability, is the exact conceptualisation of Web accessibility (Hong, Trimi, Kim, \& Hyun, 2015; Li, Yen, Lu, \& Lin, 2012b).

From a broad perspective, for a website to be considered accessible it must comply with existing regulations and guidelines. The World Wide Web Consortium (W3C), besides being considered one of the most relevant institutions in what concerns the Web related issues, has created a set of Web accessibility guidelines designed to help Web content developers to produce accessible content (Lazar, Dudley-Sponaugle, \& Greenidge, 2004). According to the referred guidelines, all Web content must be entirely 
perceivable, easy to operate, simple to understand and robust enough to be compatible with several agents and assistive technologies. Hence, for Web platform developers and Web content creators to be able to achieve proper levels of accessibility, they may choose from two separate approaches. The first available path is considered as more functional given that it focuses its attention on users' limitations and on the viable solutions that can present an answer to those limitations. The second approach is a more technical one, given that it concentrates its efforts on the existing Web technology uses and customisations as paths to decrease the impact of the obstacles triggered by difficult uses and to correct interaction with the existing Web content (Gilbertson \& Machin, 2012).

Even though the importance of delivering accessible content to the users has been proved, websites are yet lacking the necessary compliance with the existing guidelines that would allow them to become accessible to all (Gonçalves, Martins, Pereira, Oliveira, \& Ferreira, 2013; Kurt, 2016; Oh \& Chen, 2015). By acknowledging this situation, several efforts have been made towards improving not only the development tools but also towards improving the Web accessibility evaluation tools, techniques and methods in order to allow an easier maintenance of the necessary compliance levels that would foster websites' universal access feature (Gambino, Pirrone, \& Giorgio, 2016; Park \& Lim, 2015; Raji et al., 2014).

According to various researchers, such as Bensley et al. (2014), the existence of eHealth websites has become very important for the overall promotion of health knowledge and for triggering the adoption of healthy behaviours, thus the need for these websites to be available to all the Web users, including those with some sort of disability. However, and despite this almost impelling need, the accessibility and usability compliance on eHealth websites is still very low and needs to be properly addressed (Martins et al., 2016). This same perspective is defended by Niu, Luo, Liu, and Xiao (2016) and Nigro, Iannuzzi, Petracca, and Del Vecchio (2015), according to whom there is a clear set of accessibility and usability issues associated with eHealth websites that must be addressed in order to ensure universal access to all. This can only be achieved if new methodologies and approaches are defined for performing more complete and focused evaluations of the levels of accessibility and usability compliance presented by the referred websites.

\section{Healthcare websites evaluation}

The users' degree of satisfaction and interest when using a given website or Web platform is directly related to their ability to be fully accessed as well as being simple and intuitive to use (Aizpurua, Arrue, \& Vigo, 2015) (Santarosa, Conforto, \& Machado, 2014). To a certain point, this concern should not be a current concern, given the existence of several sets of guidelines and standards which regulate the implementation of accessibility and usability features on websites, and given the progress in Web related technologies. These Web related technologies, in their majority, if they are properly used can support the creation and development of accessible and usable Web content. However, as one can easily observe by performing a simple and straightforward analysis to existing scientific literature, several research records state that the majority of available websites do not present the necessary compliance with the existing accessibility and usability standards and guidelines (Gambino et al., 2016; Gonçalves et al., 2015; Gonçalves, Martins, Pereira, et al., 2013; Parmanto \& Zeng, 2005).

Despite these issues, one might think, from the perspective of authors such as Li, Yen, Lu, and Lin (2012a); Pereira, Ferreira, and Archambault (2015), one of the main causes for the existence of
Web accessibility and usability issues is the complexity associated with the existing standards and guidelines that increase the difficulty associated with developing websites that meet the referred regulations. As a result, the majority of the websites' accessibility and usability issues are still to be resolved and as we witness the development and adoption of new and more advanced ICT the more critical it becomes for websites to deliver to their users a universal access warranty (hence ensuring all users, including those with some sort of disability or incapacity, an easy access to the available content) (Snaprud \& Sawicka, 2007; Zhang et al., 2015).

With our eye on identifying the reality behind the accessibility levels of the Iberian health care institutions' websites, a research project has been planned and executed for a period of two years during which a list of Portuguese and Spanish healthcare institutions' websites were assessed against a Web accessibility evaluation platform, in order not only to reach a set of up-to-date results, but also to perceive a timeline trend based on the accessibility levels of the referred websites. Hence, two separate assessments have been performed (Fig. 1): a first one at the beginning of 2015 and a second one during the first trimester of 2016, where only 10 organisations with the best and worst results from both countries were analysed.

\subsection{Automatic web accessibility evaluation}

The evaluation procedure that supported each of the evaluation procedures has been composed during three stages: 1) target group definition and analysis; 2) target group websites evaluation with ACCESSWEB against WCAG 2.0; and 3) results treatment, analysis and discussion. As presented on the initial publication with the scope of the present research project (Martins et al., 2016), the Iberian Peninsula development in terms of Internet use is very

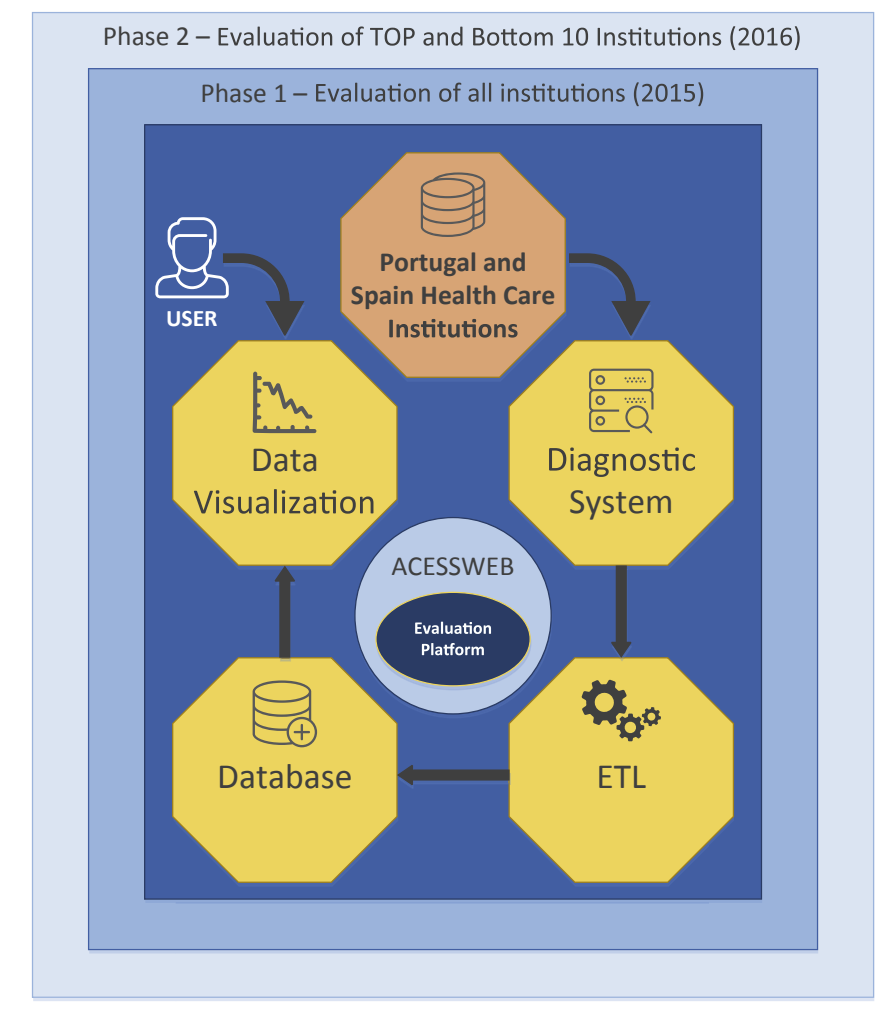

Fig. 1. Visual representation of the two evaluation phases performed to reach indicators on Iberian eHealth accessibility. 
considerable and translates into a very common use of its features to attain information and communicate. According to Walsh, Hamilton, White, and Hyde (2015) and Mano (2015), seeking for health information online has become a common practice, particularly for those communities who incorporated the Internet as part of their daily lives. With this in mind, and following Chang, Pang, Tarn, Liu, and Yen (2015) and J. Lee et al. (2016), we bring arguments in favour of the intentions demonstrated by the Internet users regarding the use of healthcare institutions websites. This use generally is not only aimed at communicating with their institution but also at obtaining health related knowledge (Ow, Wetherell, Papa, Bolton, \& Lawrentschuk, 2015). Thus, a decision has been made to perceive whether the Iberian health care institutions websites were accessible to all the Iberian citizens.

To perform the referred evaluations, the ACCESSWEB platform was used because of its ability to automatically evaluate the full extension of a website against WCAG 2.0 and present the evaluation results in a structured, simple and very usable manner. There is a wide variety of software and online services that can check whether a website complies with the Web accessibility and guidelines. The ACCESSWEB platform developed by the research team has the ability to perform multiple evaluations simultaneously based on different guidelines (i.e.: WCAG 1.0, WCAG 2.0, Section 508), which is a necessary condition to conduct large-scale studies in a short time when the availability of human and financial resources is reduced. The ACCESSWEB platform is composed of different technologies and software, organised in layers, wherein each is responsible for well-defined functions. 1) The diagnostic layer performs the analysis of accessibility and usability to websites with a list of web addresses, previously stored in a shared database, which also serves as a repository of the test results. The analyses run in parallel on a cluster of virtual machines. 2) The analytics layer processes the data obtained using techniques of Extract, Transform and Load (ETL) and Data Visualisation to produce the different indicators presented in this and other studies carried out by the research team. 3) The layer website deals with the presentation of the study results in the form of dashboards displaying the evaluations' results in a structured manner. In order for the results analysis to be significantly easier and rapid, the ACESSWEB also allows users to create their own data analysis schemas and inherent visual dashboards (Gonçalves et al., 2015).

\subsection{Iberian ehealth websites accessibility indicators}

The initially performed Web accessibility evaluation to the Iberian health care organisations websites focused on an initial list of 1098 entities (directly supplied by both the Portuguese and Spanish governmental entities responsible for regulating the healthcare sector) (Ministerio de Sanidad, 2015; PORTUGAL, 2015; SNS, 2015) and after a detailed analysis it resulted in a final set of 697 online and active websites (Martins et al., 2016). One of the first interesting acknowledgements that was possible to achieve was the fact that 140 of the referred organisations shared a website with one or more organisations and that despite the importance of having an online presence, almost 250 organisations didn't have a website or had one that was offline (Fig. 2). This result shows that against all arguments presented by authors such as Huang, Wang, and Liu (2014) and Kelly et al. (2015), and according to whom the existence of a website associated with a healthcare organisation is very important for their patients to be able to create a more trustful relation and acquire the necessary knowledge to live a healthier life, more than $20 \%$ of the Iberian healthcare organisations do not have any type of concern about their online presence or about the impact that this presence might have on their patients' life.

After performing the accessibility evaluation and treating the

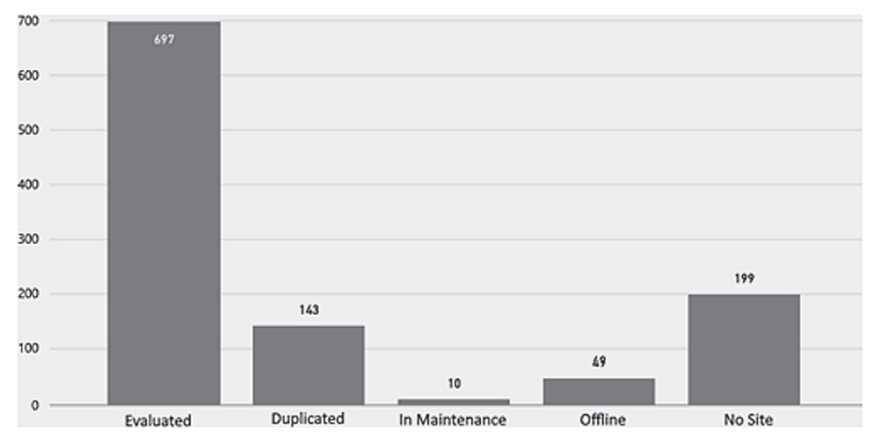

Fig. 2. Initial evaluation (2015) - Target group analysis from an Iberian Peninsula Perspective. Adapted from (Martins et al., 2016).

achieved results another very interesting consideration has been reached. Within the almost 700 evaluated websites there wasn't a single WCAG 2.0 compliant website, meaning none of the websites ensured a universal access. As a result of this poor result and aiming at understanding in a more detailed manner how the Iberian eHealth websites behaved against the WCAG 2.0 guidelines, an analysis to the achieved results has been made and an accessibility score ( $\sum$ Errors $)$ hements $)$ has been reached. This score, calculated according to the indications presented by Gonçalves et al. (2015), represents a direct relation between the number of detected accessibility errors and the number of elements that are included in the website being evaluated. Hence, the smaller the accessibility score the more accessible a website is. In Fig. 3 one can observe the distribution of the referred accessibility score according to the three defined perspectives (Iberian Peninsula, Portugal and Spain).

When analysing Fig. 3, one can perceive that a significant part of the evaluated Portuguese eHealth websites has an accessibility score bigger than 1 , thus indicating that they have more than 1 error for each website element. Another important fact that one can acknowledge is that only $4.3 \%$ of the Portuguese sample had a score inferior to 0,1, meaning that only a very small set of the Portuguese healthcare organisations present a website that ensures all the citizens, including those with some sort of disability or incapacity, an easy access to the available content. On the other hand, and after considering the Web accessibility score results achieved by the Spanish healthcare organisations, one can easily understand that almost $50 \%$ of the evaluation websites have a score equal or inferior to 0.3 , hence indicating the existence of important concerns regarding the accessibility topic and the incorporation of accessibility features in the referred websites.

Given the limited conclusions drawn from the initial evaluation and especially because of the poor accessibility levels presented by means of the evaluated websites, the research team sent a notification to each evaluated organisation indicating not only the focus of the performed study but also their particular results. This effort has been made aiming at increasing the organisations' awareness on the topic and consequently at improving the overall accessibility of the Iberian eHealth websites. In order to assess how the referred effort resulted in a real improvement of the evaluated websites, a new evaluation activity was performed. In order to allow websites managers to perform the necessary corrections, changes and optimizations to their websites, and following the argument that typical software projects have a duration of 100-man days (Lalsing, Kishnah, \& Pudaruth, 2012), a period of three months has been given before starting this second evaluation procedure. This time the focus was placed on the 10 websites from both Portugal and Spain which have obtained the best and the worst results during the initial evaluation. Hence, a total of 40 websites were evaluated, 

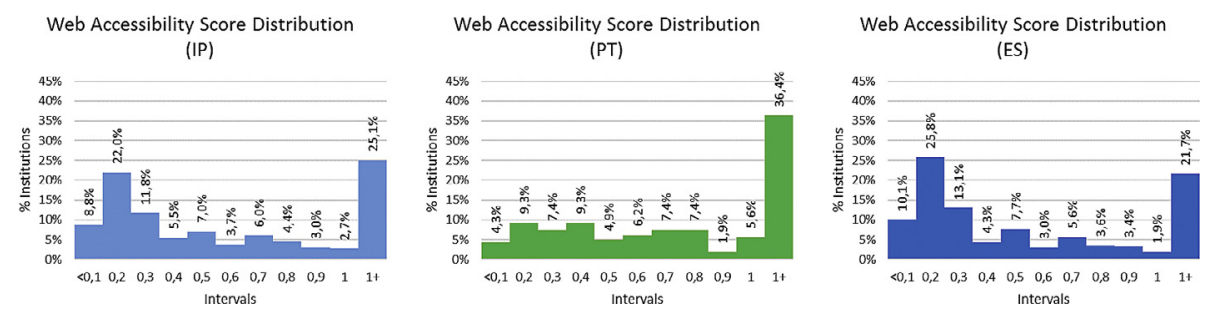

Fig. 3. Initial evaluation Web accessibility score distribution. Adapted from (Martins et al., 2016).

using once again the ACCESSWEB platform (Gonçalves et al., 2015) and WCAG 2.0 guidelines as the technical regulation.

As one can perceive in Table 1, each country evolved in a particular manner. In the Portuguese case, the accessibility score of the 2015 top 10 websites has increased (indicating an equivalent increase in the number of identified accessibility errors), in the same manner as the average number of elements in each website has risen. This particular fact allows us to understand that despite dedicating the time and effort to introduce new features, the concerns towards the accessibility topic were diminished and as a consequence the ratio of detected errors per element has increased. For the Spanish side, despite a significant decrease in the average number of evaluated elements per website, the average accessibility score has increased by a very considerable amount. This issue might be considered as an indicator that the maintenance tasks performed on the websites have resulted in new accessibility errors, instead of fixing the already existing ones.

On the other side of the spectrum, when analysing the comparison between the two sets of results accomplished for the 2015 ten worst websites (Table 2), one can easily perceive that during the time between the evaluations procedures, a decisive effort has been taken in order not only to correct the previously detected accessibility errors but also to develop even further their websites and to include new elements. From a scientific perspective, this might indicate that the existing efforts towards creating a global awareness on the topic and towards understanding the difficulties behind the development of accessible Web content are finally translating into real life changes. From an ethical and social point of view we believe that these results show that organisations are starting to introduce Web accessibility features in the developed Web content.

Despite the interesting results from the second evaluation stage, the research team assumed that it would be very interesting to understand which accessibility errors had the biggest impact on the overall accessibility compliance of the target group. This analysis, mirrored in Table 3, allows to understand that the top 5 errors are not technically complex and could easily be fixed. This fact indicates what the existing literature already claims (Gonçalves, Martins, Branco, et al., 2013; Gonçalves, Martins, Pereira, et al., 2013): The Web accessibility issue is mostly a problem of awareness and will, hence not a technical or functional matter.

Table 1

Average accessibility score and average number of elements evaluation per site for the top 10 best websites from the 2015 evaluation.

\begin{tabular}{llll}
\hline \multicolumn{2}{l}{ Portugal, 2015 eHealth Top 10 Websites } \\
\hline Country & Year & Avg. accessibility score & Avg. elements per website \\
\hline Portugal & 2015 & $8,01 \%$ & 12.267 \\
Portugal & 2016 & $8,47 \%$ & 12.854 \\
Spain & 2015 & $3,03 \%$ & 19.590 \\
Spain & 2016 & $4,15 \%$ & 15.430 \\
\hline
\end{tabular}

Table 2

Average accessibility score and average number of elements evaluation per site for the top 10 worst websites from the 2015 evaluation.

\begin{tabular}{llll}
\hline \multicolumn{2}{l}{ Portugal, 2015 eHealth Bottom 10 Websites } \\
\hline Country & Year & Avg. accessibility score & Avg. elements per website \\
\hline Portugal & 2015 & $392,36 \%$ & 103 \\
Portugal & 2016 & $109,48 \%$ & 391 \\
Spain & 2015 & $505,56 \%$ & 98 \\
Spain & 2016 & $362,85 \%$ & 133 \\
\hline
\end{tabular}

\section{Web accessibility and usability evaluation procedure}

Existing scientific literature demonstrates that the most effective manner of assessing the accessibility compliance of a given website is combining both automatic tools and manual evaluation procedures. Automatic tools are effective at identifying accessibility errors but not on a level comparable to what an expert human user can achieve. This issue can lead to the persistence of accessibility errors, even when tools indicate that everything is compliant. For this reason, automatic tools should not be a substitute of a manual evaluation but act as a complement in a complete evaluation procedure (Gohin \& Vinod, 2013; K.; Lee, Choi, \& Shin, 2013).

The development and maintenance of accessible Web content is deeply dependent on the level of knowledge on Web accessibility and usability that developers and creators have. Despite the availability of considerable amounts of both scientific and technical information that could help developers learn how to implement and maintain their work accessible, these resources are not easy to understand or to apply (Freeman, 2013; Schmutz, Sonderegger, \& Sauer, 2016; Vazquez, 2014).

Through the analysis of the existing research and standards/ guidelines, and its combination with the results achieved both by an initially performed study on the eHealth websites accessibility compliance (Martins et al., 2016) and by a new set of results on the Iberian eHealth websites WCAG 2.0 compliance, a proposal for a full scope Web accessibility and usability evaluation procedure is presented as a solution for the existing accessibility issues. This procedure is composed of three perspectives: (1) automatic Web accessibility evaluation; 2) manual Web accessibility evaluation; and 3) Web Usability Heuristics Evaluation) that, when combined, will deliver more accurate and useful results on the possible accessibility and usability issues that might exist and on the easier manner to fix them. In what concerns the applicability of the referred evaluation procedure, it should start with an automatic evaluation activity responsible for crawling the entire website and assess all its elements against the WCAG 2.0 guidelines. A manual accessibility and usability evaluation should then be performed in order to not only validate the automatic procedure results but also to ensure the absence of usability constraints or faults. 
Table 3

Variance between the top 5 errors with the most occurrences during the 2015 and 2016 Portuguese evaluations.

\begin{tabular}{lc}
\hline Portugal eHealth websites top 5 errors & Occurrences \\
\hline Error & 640 \\
\hline $\mathbf{2 0 1 5}$ & 576 \\
Identify row and column in data tables using TH elements. & 522 \\
Using ALT text which duplicates link text in the same link. & 522 \\
All ONMOUSEOUT handlers should have an equivalent ONBLUR handler. & 521 \\
All ONMOUSEOVER handlers should have an equivalent ONFOCUS handler. & 640 \\
Use relative rather than absolute units in CSS property values & 522 \\
$\mathbf{2 0 1 6}$ & 522 \\
Identify row and column in data tables using TH elements. & 576 \\
All ONMOUSEOUT handlers should have an equivalent ONBLUR handler. & 521 \\
All ONMOUSEOVER handlers should have an equivalent ONFOCUS handler. & \\
Using ALT text which duplicates link text in the same link. & \\
Use relative rather than absolute units in CSS property values &
\end{tabular}

\subsection{Web accessibility automatic evaluation}

According to W3C (2008) and Gonçalves, Martins, Pereira, et al. (2013), the automatic Web accessibility evaluation procedure must encompass three different concerns: 1) evaluation scope definition; 2 ) evaluation tools identification; and 3) definition of the results format and presentation. By following these indications prior to performing the evaluation task one makes sure that the achieved results will be coherent and valid.

\subsubsection{Evaluation scope definition}

In order to define the Web accessibility evaluation scope, one must identify the evaluation criteria that are going to be used as the technical basis of the evaluation and then characterise the intended target group. Considering the ambition that one must have to obtain the most accurate and foolproof results, we believe that choosing an international standard, regulation or recommendation is the best option. Proper examples of such technical support elements are the W3Cs Web Accessibility Guidelines 2.0 (AAA level of compliance) and the USA Government Section 508.

For the target group characterisation, the evaluation managers should during an initial stage make an effort to understand what is their goal (Ex: evaluate one website or evaluate a set of websites), followed by an analysis towards the availability of the chosen websites and their technical specificities (Ex: coding language and technology, existence of login, etc.).

\subsubsection{Evaluation tools identification}

Even though there is not an evaluation tool considered to be the best, or to be indicated for the job, by all national and international organisations, the act of choosing solely depends on the evaluation managers, because they are the ones who must find the most adequate tool for their evaluation goals. From our perspective and experience, the selection of the tool should be made according to the following set of features: 1 ) ability to automatically evaluate the entire website or a user-defined number of pages; 2 ) be available in, at least, a universal language (Ex: English); 3) ability to evaluate a website against international standards or regulations (Ex: WCAG 2.0 or Section 508); and 4) present the evaluation results in a simple and easy to analyse manner.

In order to ease the choice of the Web accessibility evaluation tool, W3C has published a list (W3C, 2016) with several tools that have the ability to assess a website or a webpage against international Web accessibility standards or regulations.

\subsubsection{Definition of results format and presentation}

The information extracted from the automatic evaluation procedure is very important because it holds the knowledge that researchers seek and the indicators regarding what is right and what is wrongly developed. By analysing the existing literature (Fernandes et al., 2012; Gonçalves, Martins, Pereira, et al., 2013; K.; Lee et al., 2013; Martins et al., 2015), we notice that the most common format used to analyse and interpret the evaluations results are the statistical indicators, mainly gathered in tables and charts that allow not only to understand the overall results but also to quickly and easily perceive the target group tendency towards being (or not) Web accessible compliant.

\subsection{Web accessibility manual evaluation}

The manual evaluation activity is, as one can understand, extremely difficult to implement when evaluating a target group with a considerable size. Nevertheless, when evaluating a number of websites that allow the manual assessment to be feasible, the evaluation managers should use accessibility experts to, in a first phase, confirm all the errors reported by the automatic evaluation tool (these can generate false positives and negatives) and, in a second phase, evaluate the entire site manually through the use of assistive technologies with all types of users, including those with disabilities (from which one can expect a more interesting feedback) (T. Rocha et al., 2015).

\subsection{Web usability evaluation}

As stated by Nielsen (1994), usability can be perceived as a quality factor associated with all the existing software. The same author also argues that the referred concept is not closed but continuously evolving around a set of principles: learnability, efficiency, memorability, few errors and user satisfaction.

In regards to accessibility, Lew, Olsina, Becker, and Zhang (2012) and Matera, Rizzo, and Carughi (2006, pp. 143-180) state that it should be interpreted as an extension and complementation to the usability conceptualisation, as it enforces the basis for users to perform website related tasks with effectiveness, with a high level of acquired knowledge, while addressing, at the same time, the use of a given web content in a specific context. "The usability of web content puts its focus on fewer errors occurring; the accessibility of web content aims for it to be used and accessed by everyone" (Gonçalves, Martins, Pereira, et al., 2013).

\subsubsection{Heuristics evaluation}

After assessing the accessibility levels of the target group, a usability evaluation should be performed. The present proposal holds that the most adequate technique to be used first for assessing the websites usability should be a heuristics evaluation. Examples of heuristics for assessing the usability of a website can 
be found throughout the scientific and technical literature, and especially the works of Alotaibi (2013), Pearrow (2006), Krug (2005) and Nielsen (1994) are particularly complete and simple to interpret.

In order to perform a heuristic assessment, the evaluation managers should aim at collecting two types of data: 1) quantitative - number of non-compliance issues for each heuristic, their locations (URL) and the severity of the identified errors; and 2) qualitative - comments made by the heuristics evaluators (experts on the field of usability). Considering the possible complexity of a website, the heuristics evaluation should focus its attention on the set of webpages that better represent the typical user interaction with the website.

\section{Final considerations and conclusions}

\subsection{Practical and theoretical implications}

Web accessibility has acquired an increased relevance as a result of the constant growth registered with regards to Internet access and to the variety of devices used to access the Web (Ex: computers, notebooks, smartphones, tablets and smart watches). The current state of science and technology has created the necessary conditions for humans to live longer and with better health related conditions. Hence, it is clear that the number of users needing to be granted access to all sorts of software and ICT is growing, apart from the current ratios of users with some sort of disability. The existence of Web accessibility features should allow a direct access for all the Internet users, including those with some sort of disability, who might need to interact with existing Web content and feel included in the society in which we live.

Despite the observations presented above, the existing laws and guidelines and the numerous efforts made by several international organisations, the Web accessibility topic has not been considered to be a critical issue for the development and maintenance of the Web platforms that are so important for both organisations and users. The parts involved seem unaware of the real difficulties that the Web users have today, and certainly unaware of those problems that the future holds.

Hence, from a theoretical perspective the proposed full scope Web accessibility and usability procedure represents a solid contribution to science given that it is supported by a vast experience in the field and encompasses the existing best practices. Thus, the referred proposal has even the potential to become a reference in the field of expertise and to be used by individual users, web developers, organisations and researchers.

The presented results inherent to the accessibility evaluation of the Iberian eHealth websites might also contribute to an increased concern about the topic and raise awareness of all the actors involved in planning, developing and exploring Web platforms and websites. This is a critical issue because, as proved by the concerning number increase of accessibility detected on the evaluated websites during a one-year timeline, the Web accessibility related concerns, presented by the owners of the evaluated websites, had an insignificant importance.

\subsection{Limitations and future work}

The presented research aimed at reaching a timeline perspective on the Web accessibility levels of the Iberian eHealth websites, represented by the websites owned by the Portuguese and Spanish health care institutions. Despite reaching this goal, and being able to identify some acknowledgements, in line with other research (Oliveira \& Martins, 2010a, 2010b), we believe that studying the referred issue using a broader target group would allow us to reach more interesting results.

Another concern that from our perspective would improve the overall impact of the research project was the inclusion of manual evaluation activities as complements of the performed automatic evaluations (T. Rocha et al., 2015). This would not only help confirm the results already achieved, but would also allow us to present a real example of how to use the proposed evaluation procedure.

\subsection{Conclusions}

As Internet becomes one of the most adopted technologies of all times and users start to take advantage of its global dynamics to reach and share health related information, the global awareness of the topic is also increasing and, as a consequence, will have a positive impact on the prevention and early detection of diseases, hence having a relevant impact on the population's quality of life. This realisation is, from our perspective, very significant given that it highlights the relevance of having the referred health related information accessible to all the users, including those with some sort of disability or impairment. Hence, the inherent need for the websites to host the referred information and to be compliant with existing Web accessibility and usability guidelines and ensure universal access to its content.

The existing literature about the development of accessible websites and Web platforms highlights that the current levels of compliance with Web accessibility and usability standards and guidelines is somewhat poor and that this issue is mostly due to two different problems: 1) the complexity behind existing standards that makes it hard for developers and managers to understand them; and 2) the lack of knowledge (and possibly) interest demonstrated by developers and project managers to incorporate accessibility related techniques during the specification and development stages. As a result of these issues, the number of totally accessible and usable websites is considerably low and the concept of universal access can only be applied to the Web as a future goal.

In line with the arguments presented above and the significant amount of dispersed information on how to create accessible Web content, an evaluation to the Iberian eHealth websites allowed us to observe that the majority of these websites are very far from having the necessary features that would allow all the users, including those with some sort of disability, to use them. This issue is not only extremely concerning from an ethical perspective but mainly from a public health point of view, given that several millions of users (just in Portugal and Spain alone) are being denied access to information that could, in other way, improve their lives.

As a consequence of merging the achieved Iberian eHealth accessibility poor results with an extensive background on assessing Web accessibility and usability, a full scope Web accessibility and usability evaluation procedure has been proposed. This evaluation procedure assumes itself as a significant contribution given that it will not only contemplate both automatic and manual evaluation activities, (hence being in line with existing international standards and guidelines), but it will also give details in such a manner so that it can be easily used by (health care) organisations or Web content developers.

\section{Acknowledgments}

This paper was supported by project ACESSWEB, financed by the Portuguese national funding agency for science, research and technology, with a total financial support of $50.000 €$ and a twelvemonth time-frame for execution. 


\section{References}

Aizpurua, A., Arrue, M., \& Vigo, M. (2015). Prejudices, memories, expectations and confidence influence experienced accessibility on the Web. Computers in $\mathrm{Hu}$ man Behavior, 51, 152-160.

Alotaibi, M. (2013). Assessing the usability of university websites in Saudi Arabia: A heuristic evaluation approach. In Information Technology: New generations (ITNG), 2013 tenth international conference (pp. 138-142). IEEE.

Bensley, R., Hovis, A., Horton, K., Loyo, J., Bensley, K., Phillips, D., et al. (2014). Accessibility and preferred use of online Web applications among WIC participants with Internet access. Journal of Nutrition Education and Behavior, 46(3), S87-S92.

Chang, M., Pang, C., Tarn, J., Liu, T., \& Yen, D. (2015). Exploring user acceptance of an e-hospital service: An empirical study in Taiwan. Computer Standards \& Interfaces, 38, 35-43.

Domenichiello, M. (2015). State of the art in adoption of e-Health services in Italy in the context of European Union E-Government strategies. Procedia Economic and Finance, 23, 1110-1118.

EC.. (2014). Europeans becoming enthusiastic users of online health information in Digital Economy \& Society. European Commission.

Eysenbach, G. (2001). What is e-health? Journal of medical Internet research, 3(2), e20.

Fernandes, N., Costa, D., Duarte, C., \& Carriço, L. (2012). Evaluating the accessibility of web applications. Procedia Computer Science, 14, 28-35.

FRA.. (2015). European union agency for fundamental rights - people with disabilities in (vol. 2015).

Freeman, M. (2013). Factors influencing webmasters and the level of web accessibility and section 508 compliance at SACS accredited postsecondary institutions: A study using the theory of planned behavior. ERIC.

Gambino, O., Pirrone, R., \& Giorgio, F. (2016). Accessibility of the Italian institutional web pages: A survey on the compliance of the Italian public administration web pages to the Stanca act and its 22 technical requirements for web accessibility. Universal Access in the Information Society, 15(2), 305-312.

Gilbertson, T., \& Machin, C. (2012). Guidelines, icons and marketable skills: An accessibility evaluation of 100 web development company homepages. In Proceedings of the international cross-disciplinary conference on web accessibility (p. 17). ACM.

Gohin, B., \& Vinod, V. (2013). A Study on web accessibility in perspective of evaluation tools. International Review on Computers and Software (IRECOS), 8(11) 2648-2654.

Gonçalves, R., Martins, J., Branco, F., \& Barroso, J. (2013). Web accessibility-from the evaluation and analysis to the implementation-the anoGov/PEPPOL case. In Universal access in human-computer interaction. User and context diversity (pp. 664-673). Springer.

Gonçalves, R., Martins, J., Branco, F., Pereira, J., Rocha, T., \& Peixoto, C. (2015) Accessweb barometer - a web accessibility evaluation and analysis platform. In INTERNET 2015-the seventh international conference on evolving Internet. Malta: IARIA.

Gonçalves, R., Martins, J., Pereira, J., Oliveira, M., \& Ferreira, J. (2013). Enterprise Web accessibility levels amongst the Forbes 250: Where art thou o virtuous leader? Journal of business ethics, 113(2), 363-375.

Hakim, L., \& Deswindi, L. (2015). Assessing the effects of e-servicescape on customer intention: A study on the hospital websites in South Jakarta. Procedia - Socia and Behavioral Sciences, 169, 227-239.

de Haydu, C., Eleswarapu, S. V., Dabaja, A. A., \& Duke, C. M. (2015). Objective assessment of the oncofertility educational information, available to women, on the websites of $\mathrm{NCI}$-designated cancer centers in the US: Do socioeconomic demographic profiles by state make a difference? Fertility and Sterility, 104(3), e262.

Hong, S., Trimi, S., Kim, D., \& Hyun, J. (2015). A Delphi study of factors hindering web accessibility for persons with disabilities. Journal of Computer Information Systems, 55(4), 28-34.

Huang, E., Liu, T., \& Wang, J. (2014). E-health videos on Chinese hospitals' websites. International Journal of Healthcare Management, 7(4), 273-280.

Huang, E., Wang, J., \& Liu, T. (2014). Interactive e-health tools for patients on Chinese hospitals' websites. International Journal of Healthcare Management, 7(2) $75-83$.

Hwang, J., McMillan, S., \& Lee, G. (2003). Corporate web sites as advertising: An analysis of function, audience, and message strategy. Journal of Interactive Advertising, 3(2), 10-23.

Kelly, L., Ziebland, S., \& Jenkinson, C. (2015). Measuring the effects of online health information: Scale validation for the e-Health Impact Questionnaire. Patient Education and Counseling, 98(11), 1418-1424.

Klein, E., Bolfing, A., \& Riesch, M. (2014). Checking web accessibility with the content accessibility checker (CAC). In K. Miesenberger, D. Fels, D. Archambault, P. Peňáz, \& W. Zagler (Eds.), Computers helping people with special needs (Vol 8547, pp. 109-112). Springer International Publishing.

Krug, S. (2005). Don't make me think: A common sense approach to web usability. Pearson Education India.

Kurt, S. (2016). Accessibility of Turkish university web sites. Universal Access in the Information Society, 1-11.

Lalsing, V., Kishnah, S., \& Pudaruth, S. (2012). People factors in agile software development and project management. International Journal of Software Engineering \& Applications, 3(1), 117.
Lazar, J., Dudley-Sponaugle, A., \& Greenidge, K. (2004). Improving web accessibility: A study of webmaster perceptions. Computers in Human Behavior, 20(2), 269-288.

Lee, K., Choi, K., \& Shin, H. (2013). Web accessibility evaluation of community rehabilitation center homepage. Journal of Convergence Information Technology, $8(12), 373$

Lee, J., Choudhry, N., Wu, A., Matlin, O., Brennan, T., \& Shrank, W. (2016). Patient use of Email, Facebook, and physician websites to communicate with physicians: A national online survey of retail pharmacy users. Journal of General Internal Medicine, 31(1), 45-51.

Leite, P., Gonçalves, J., Teixeira, P., \& Rocha, Á. (2014). Towards a model for the measurement of data quality in websites. New Review of Hypermedia and Multimedia, 20(4), 301-316.

Lew, P., Olsina, L., Becker, P., \& Zhang, L. (2012). An integrated strategy to systematically understand and manage quality in use for web applications. Requirements Engineering, 17(4), 299-330.

Lin, C., Wittevrongel, L., Moore, L., Beaty, B., \& Ross, S. (2005). An Internet-based patient-provider communication system: Randomized controlled trial. Journal of Medical Internet Research, 7(4).

Li, S., Yen, D., Lu, W., \& Lin, T. (2012a). Migrating from WCAG 1.0 to WCAG 2.0-a comparative study based on web content accessibility guidelines in Taiwan. Computers in Human Behavior, 28(1), 87-96.

Li, S., Yen, D., Lu, W., \& Lin, T. (2012b). Migrating from WCAG 1.0 to WCAG 2.0 - a comparative study based on web content accessibility guidelines in Taiwan. Computers in Human Behavior, 28(1), 87-96.

Mano, R. (2015). Online health information, situational effects and health changes among e-patients in Israel: A 'push/pull'perspective. Health Expectations, 18(6), 2489-2500.

Martins, J., Barroso, J., Gonçalves, R., Sousa, A., Bacelar, M., \& Paredes, H. (2015). Transforming e-procurement platforms for PEPPOL and WCAG 2.0 compliance. In Information science and applications (pp. 973-980). Springer.

Martins, J., Gonçalves, R., Branco, F., Pereira, J., Peixoto, C., \& Rocha, T. (2016). How Ill Is online health care? an overview on the iberia peninsula health care institutions websites accessibility levels. In New advances in information systems and technologies (pp. 391-400). Springer.

Matera, M., Rizzo, F., \& Carughi, G. (2006). Web usability: Principles and evaluation methods. In Web engineering. Springer.

Ministerio de Sanidad, S. S. e. I. (2015). Centros y Servicios del Sistema Nacional de Salud. http://www.msssi.gob.es/ciudadanos/prestaciones/centrosServiciosSNS/ home.htm.

Naoui, F. \& Zaiem, I. (2015). Reasons for consumer trust in health websites: An approach integrating website-based factors and personality-based factors. International Journal of Management Sciences and Business Research, 4(2), 28-39.

Nielsen, J. (1994). Usability engineering. Elsevier.

Nigro, C., Iannuzzi, E., Petracca, M., \& Del Vecchio, S. (2015). How Health Care Moves on the Web: The Case of Health Social Network. In Toulon-verona conference" excellence in services".

Niu, L., Luo, D., Liu, Y., \& Xiao, S. (2016). The accessibility, usability, and reliability of chinese web-based information on HIV/AIDS. International Journal of Environmental Research and Public Health, 13(8), 834.

Noh, K., Jeong, E., You, Y., Moon, S., \& Kang, M. (2015). A study on the current status and strategies for improvement of web accessibility compliance of public institutions. Journal of Open Innovation: Technology, Market, and Complexity, 1(1), $1-17$.

Oh, L., \& Chen, J. (2015). Determinants of employees' intention to exert pressure on firms to engage in web accessibility. Behaviour \& Information Technology, 34(2), $108-118$.

Oliveira, T., \& Martins, M. (2010a). Firms patterns of e-business adoption: Evidence for the European Union-27.

Oliveira, T., \& Martins, M. (2010b). Understanding e-business adoption across industries in European countries. Industrial Management \& Data Systems, 110(9), 1337-1354.

Ow, D., Wetherell, D., Papa, N., Bolton, D., \& Lawrentschuk, N. (2015). Patients' perspectives of accessibility and digital delivery of factual content provided by official medical and surgical specialty society websites: A qualitative assessment. Interactive journal of medical research, 4(1).

Park, E., \& Lim, H. (2015). A study on improvement of evaluation method on web accessibility automatic evaluation tool's $<$ IMG $>$ alternative texts based on OCR. Advanced Science and Technology Letters, 113, 162-166.

Parmanto, B., \& Zeng, X. (2005). Metric for Web accessibility evaluation. Journal of the American Society for Information Science and Technology, 56(13), 1394-1404.

Pearrow, M. (2006). Web site usability handbook (Internet series). Charles River Media, Inc.

Pereira, L., Ferreira, S., \& Archambault, D. (2015). Preliminary web accessibility evaluation method through the identification of critical items with the participation of visually impaired users. In C. Velasco, G. Weber, J. Barroso, Y. Mohamad, \& H. Paredes (Eds.), Proceedings of the 6th international conference on software development and technologies for enhancing accessibility and fighting info-exclusion (Vol. 67, pp. 77-86). Amsterdam: Elsevier Science Bv.

PORTUGAL, M. H. C. (2015). Health care institutions catalog. http://www.saudeportugal.pt/index.php.

PRC. (2013). Health fact sheet. Internet, Science \& Tech: Pew Research Center.

Raji, S., Mahmud, M., Tap, A. \& Abubakar, A. (2014). Usability evaluation of hospital websites in Nigeria: What affects end users' preferences?. In C. Stephanidis (Ed.), HCI international 2014-posters' extended abstracts (Vol. 435, pp. 430-434) 
Springer International Publishing.

Ralston, J., Carrell, D., Reid, R., Anderson, M., Moran, M., \& Hereford, J. (2007). Patient web services integrated with a shared medical record: Patient use and satisfaction. Journal of the American Medical Informatics Association, 14(6), 798-806.

Robeznieks, A. (2011). Online and on target. Hospitals add sophistication to their websites, improving value to patients. Modern Healthcare, 41(7), 32-33.

Rocha, Á. (2012). Framework for a global quality evaluation of a website. Online Information Review, 36(3), 374-382.

Rocha, T., Carvalho, D., Gonçalves, R., Martins, J., Branco, F., \& Bessa, M. (2015). Usability evaluation of the touch screen and mouse as input devices by people with intellectual disabilities. In P. Isaias (Ed.), 14th international conference WWW/internet 2015. Dublin, Ireland: IADIS.

Sánchez-Gordón, M., \& Moreno, L. (2014). Toward an integration of web accessibility into testing processes. Procedia Computer Science, 27(0), 281-291.

Santarosa, L., Conforto, D., \& Machado, R. (2014). Whiteboard: Synchronism, accessibility, protagonism and collective authorship for human diversity on Web 2.0. Computers in Human Behavior, 31, 591-601.

Schenker, Y., \& London, A. (2015). RIsks of imbalanced information on us hospital websites. JAMA Internal Medicine, 175(3), 441-443.

Schmutz, S., Sonderegger, A., \& Sauer, J. (2016). Implementing recommendations from web accessibility guidelines: Would they also provide benefits to nondisabled users. Human Factors: The Journal of the Human Factors and Ergonomics Society, 58(4), 611-629.

Sigala, M., \& Chalkiti, K. (2014). Investigating the exploitation of web 2.0 for knowledge management in the Greek tourism industry: An utilisation-importance analysis. Computers in Human Behavior, 30, 800-812.
Silvestre, J., Tomlinson-Hansen, S., Fosnot, J., \& Taylor, J. (2014). Plastic Surgery residency websites: A critical analysis of accessibility and content. Annals of Plastic Surgery, 72(3), 265-269.

Snaprud, M., \& Sawicka, A. (2007). Large scale web accessibility evaluation - a European perspective. Berlin: Springer-Verlag.

SNS, S.-P.d.. (2015). Entidades de Saúde. https://www.sns.gov.pt/institucional/ entidades-de-saude/.

Snyder, K., Ornes, L., \& Paulson, P. (2014). Engaging patients through your website. Journal for Healthcare Quality, 36(2), 33-38.

UN.. (2014). e-Government Survey 2014-e-Government for the future we want. United Nations.

Vazquez, S. (2014). Introducing web accessibility to localization students: Implications for a universal web. In Proceedings of the 16th international ACM SIGACCESS conference on computers \& accessibility (pp. 333-334). Rochester, New York, USA: ACM.

W3C. (2008). Web content accessibility guidelines (WCAG) 2.0 (vol. 2015). World Wide Web Consortium.

W3C. (2016). Web accessibility evaluation tools list (vol. 2016).

Walsh, A., Hamilton, K., White, K., \& Hyde, M. (2015). Use of online health information to manage children's health care: A prospective study investigating parental decisions. BMC health services research, 15(1), 1.

Wenner, C. (2008). ICT for citizens and businesses (vol. 2015). Information Society Technologies.

Zhang, M., Wang, C., Bu, J. J., Yu, Z., Zhou, Y., \& Chen, C. (2015). A sampling method based on URL clustering for fast web accessibility evaluation. Frontiers of Information Technology \& Electronic Engineering, 16(6), 449-456. 